Reporting Regulation (29 CFR Part 1904), established in 1971. Under the original reporting regulation, employers were required to collect and maintain injury and illness data and to have them available for OSHA to examine. It was determined that OSHA needed a separate provision for collection of data by mail. As such, this final rule requires employers, upon request, to report to OSHA their illness and injury data, in addition to the number of workers and the number of days worked in a designated period.

The new rule establishes a procedural mechanism for OSHA to conduct an annual survey of 10 or more employers by mail or other remote transmittal. The specific request may come directly from OSHA or its designee (eg, The National Institute of Occupational Safety and Health). The data will be used for injury and illness surveillance, to evaluate OSHA standards, and to evaluate the effectiveness of enforcement training. In addition, they will be used to direct OSHA's programs for scheduled inspections.

Concerns were expressed throughout the rule-making process that reporting this information was burdensome and duplicative and that the data could be obtained from other sources, such as the workers' compensation files. OSHA argued that this new system would provide more reliable data better suited to OSHA's needs than any available alternative. Considering that OSHA cannot directly visit the over 5 million worksites, this provides information to target their activities, including workplace inspections. OSHA reported that 80,000 workplaces were inspected in 1996.

Employers will have 30 days to submit their data after the request is received. Employers will be notified in an upcoming Federal Register notice of the type of information that needs to be collected. Much of the injury and illness information to be reported will be taken from records that employers already are required to create, maintain, and post. The employment figures to be collected are critical to OSHA's ability to evaluate the injury and illness data. This regulation was scheduled to become effective on March 13, 1997.

Occupational health and safety experts have said that this rule will benefit those employers with good health and safety programs.

FROM: Department of Labor, Occupational Safety and Health Administration. Reporting occupational injury and illness data to OSHA. Final Rule. Federal Register 62 (28) February 11, 1997:6434-6442.

\section{Safety Devices Prevent Percutaneous Injuries During Phlebotomy}

Phlebotomy, one of the most commonly performed medical procedures, has been associated with $13 \%$ to $62 \%$ of injuries reported to hospital occupational health services and with 20 of 51 documented episodes of occupationally acquired HIV infection in the United States. A collaborative study recently was conducted by the CDC and six university-affiliated hospitals located in Minneapolis, New York City, and San Francisco to evaluate safety devices used for phlebotomy. The assessment was restricted to a comparison of safety devices with conventional devices. Each hospital selected the products to be evaluated (vacuum-tube collection devices or winged steel needles with safety fea- tures). Three products were evaluated and included a resheathable winged steel needle (device 1; Safety-Lok, Becton Dickinson, Franklin Lakes, NJ); a blunt vacuumtube blood-collection needle activated while in the patient's vein (device 2; Puncture-Guard, Bio-Plexus Inc, Tolland, CT), and a vacuum-tube blood collection needle with a hinged recapping sheath (device 3; Venipuncture NeedlePro, Smith Industries-Concord Portex, Keene, NH). Each product required the healthcare worker to activate the safety feature during or after phlebotomy. During phase I of the study, hospitals used conventional phlebotomy devices and conducted enhanced surveillance for injuries (encourage reporting, newsletters and published notices, inservice training). Underreporting and estimates of number of phlebotomies performed daily was assessed with an anonymous survey. During phase II of the study, investigators replaced conventional devices with safety devices. A second survey was done and also included an assessment of satisfaction with safety devices and any adverse effects in patients.

Overall, respondents acknowledged reporting only $54 \%$ of the 564 needlestick injuries that were sustained during the previous year. The findings indicated that, for each of the safety devices evaluated, the number of phlebotomy-related percutaneous injuries was significantly less for the safety devices compared to the conventional devices (both adjusted and unadjusted for underreporting). The percentage reduction in percutaneous injury rate with safety devices was $23 \%$ for device $1 ; 76 \%$ for device 2 ; and $66 \%$ for device 3 .

The results of this study suggest that safety devices for phlebotomy may be generally acceptable to users. Activation rates of safety features and user acceptability may be influenced by factors such as the perceived risk for occupational infection by the HCW, design of the device, training provided before and after introduction of the device, length of time needed to become adept at using the device, ease of use, necessary changes in technique, and previous experience with a safety device.

FROM: Centers for Disease Control and Prevention. Evaluation of safety devices for preventing percutaneous injuries among health care workers during phlebotomy procedures-Minneapolis-St Paul, New York City, and San Francisco, 1993-1995. MMWR 1997;46:21-25.

\section{Blunt Suture Needles Reduce Risk of Percutaneous Injuries}

Percutaneous injuries (PIs) have been reported during $1 \%$ to $15 \%$ of surgical procedures, mostly associated with suturing. Most suturing is done using curved needles, although straight needles are used by some surgeons for suturing skin. Blunt suture needles (curved suture needles that have a relatively blunt tip) may be less likely to cause PIs, because they do not penetrate the skin easily. Based on a few small studies, these blunt suture needles are able to replace conventional curved suture needles for suturing many tissues, although they may require more pressure to penetrate the tissues. During March 1993 to June 1994, the CDC collaborated with three teaching hospitals in New York City to evaluate a blunt suture needle (Ethiguard, Ethicon Inc, Somerville, NJ) in gynecologic surgery. A total of 1,464 